\title{
Practical expressions for the design of laminated glass
}

\author{
Laura Galuppi $^{(1)}$, Giampiero Manara ${ }^{(2)}$, Gianni Royer Carfagni ${ }^{(1)}$ \\ ${ }^{(1)}$ Department of Civil-Environmental Engineering and Architecture, University of Parma \\ ${ }^{(2)}$ Permasteelisa Group, Vittorio Veneto, Italy
}

\begin{abstract}
Due to deformability of the polymeric interlayer, stiffness and strength of laminated glass are usually less than those corresponding to a monolith with same total thickness. A practical design tool consists in the definition of the "effective thickness", i.e., the thickness of an equivalent monolithic glass that would correspond to the same deflection and peak stress of the laminated glass, under the same constraint and load conditions. Very recently, a new model has been proposed for the evaluation of the effective thickness. Here, a comparison is made with the classical approach by Wölfel-Bennison and the new method is specialized to the most common cases of the design practice, providing synthetic tables for ease of reference and immediate applicability.
\end{abstract}

Keywords: Laminated glass, plate design, effective thickness, strength calculation, composite structures, sandwich structure.

\section{Introduction.}

An effective technique to enhance the post-glass-breakage performance of architectural glazing consists in bonding glass plies together with polymeric interlayers via lamination in autoclave at high temperature and pressure. In such a way a laminated glass acquires safety properties because, after breakage, shards remain attached to the polymer and the system maintains a small but significant load bearing capacity, avoiding injuries due to catastrophic collapse.

Stiffness and strength of laminated glass may be considerably less than those of a monolithic glass with the same total thickness, because the interlayer is unable to provide a perfect shear 
coupling. As a matter of fact, the response is affected by the shear stiffness of the polymer (in particular by its shear modulus $G$ ), that regulates the relative sliding of the constituent glass plies.

Two borderline cases can be recognized: i) the monolithic limit for $G \rightarrow \infty$, where the two glass plies are perfectly bonded together (fig. 1a) and the flexural inertia is that corresponding to the total thickness of the laminated glass; ii) the layered limit for $G \rightarrow 0$, with free-sliding plies (fig. 1b), for which the flexural inertia is the sum of the inertiae of the isolated plies. In general, the real condition is intermediate between these two borderline cases (fig. 1c).

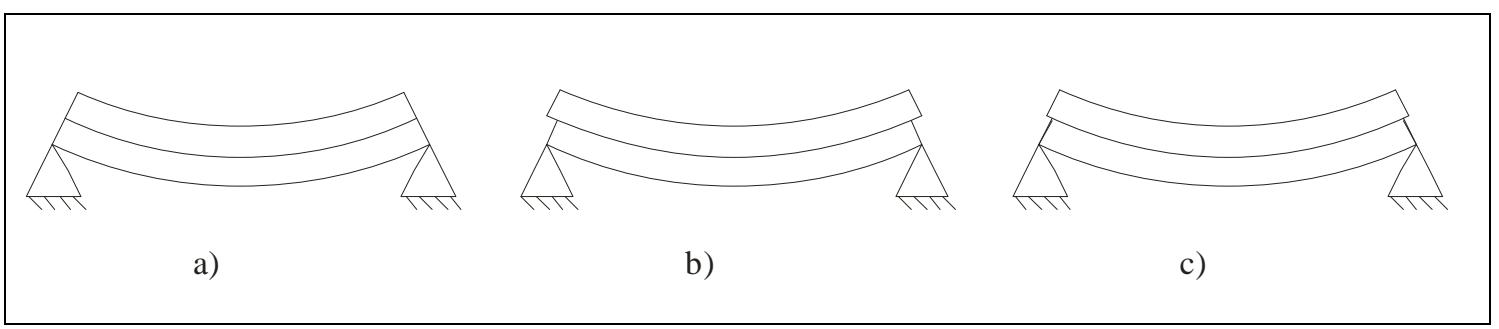

Figure 1: Laminated glass composed of two plies and one interlayer under flexure. The two limit cases of a) monolithic limit and b) layered limit; c) the intermediate configuration.

Polymers are highly viscoelastic and, consequently, their response depends upon load duration and temperature. In the design practice a full viscoelastic analysis is seldom performed, but rheological effects are taken into account by considering, for the shear modulus $G$, the secant stiffness at the end of the load history at actual room temperature. The problem is thus simplified and reduced to a case in which all the materials, including the interlayer, are considered linear elastic. Moreover, at least as a first order approximation for a preliminary design, geometric nonlinearities can be neglected when in-plane loads are absent.

In numerical computations, the response of laminated glass could be conveniently modelled by a layered shell element that takes into account the competing stiffness between glass and interlayer, but most of the commercial numerical codes do not have such elements in their library. On the other hand, a full three-dimensional analysis is complicated and time consuming. This is why, in the design practice and especially in the preliminary design, it is very useful to consider approximate methods for the calculation of laminated glass. 
Currently, the most used approach is probably that proposed by Bennison (2009) based upon the theory for composed sandwich beams proposed by Wölfel (1987)0. To illustrate, consider a laminated beam of length $l$ and width $b$ composed of two glass plies of thickness $h_{1}$ and $h_{2}$ and Young's modulus $E$, connected by a polymeric interlayer of thickness $t$ and shear modulus $G$ (fig. 2).

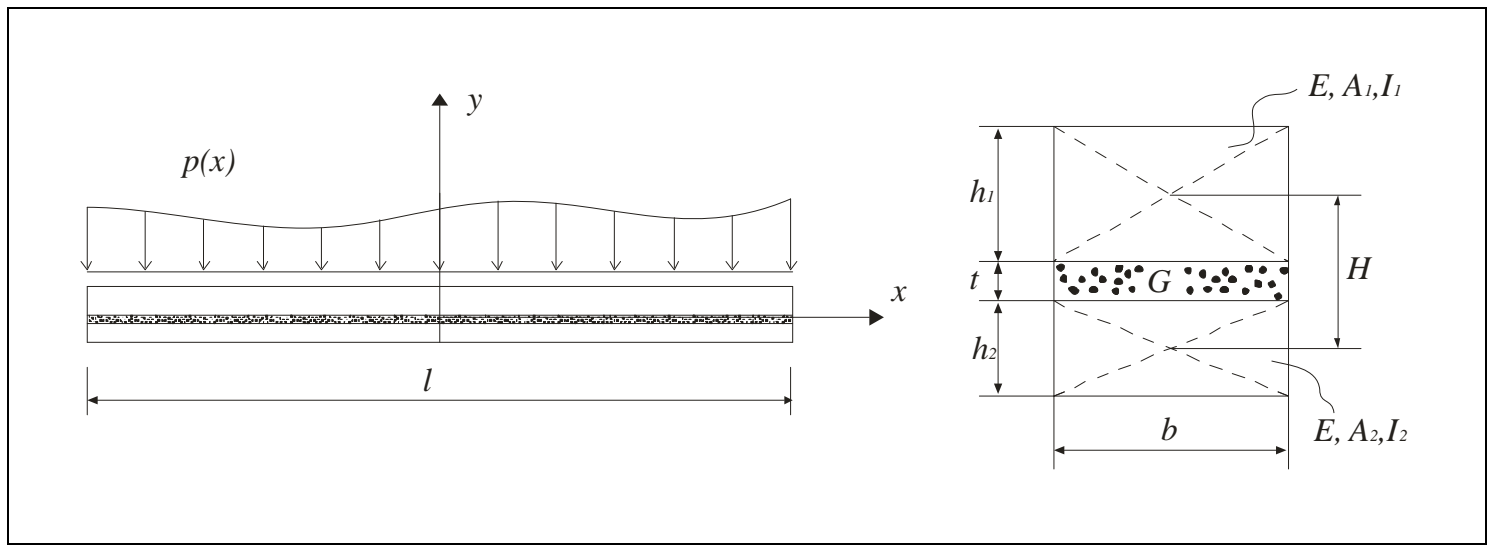

Figure 2: Beam composed of two glass plies bonded by a polymeric interlayer. Longitudinal and cross sectional view (not in the same scale).

Let

$$
A_{1}=b h_{1}, A_{2}=b h_{2}, H=t+\frac{h_{1}+h_{2}}{2}, I_{1}=\frac{b h_{1}^{3}}{12}, I_{2}=\frac{b h_{2}^{3}}{12} .
$$

When the layered limit is attained (i.e., two free-sliding glass plies), the moment of inertia of the laminated beam equals the sum $I_{1}+I_{2}$. In the monolithic limit, the moment of inertia reads

$$
I_{t o t}=I_{1}+I_{2}+\frac{A_{1} A_{2}}{A_{1}+A_{2}} H
$$

where $\frac{A_{1} A_{2}}{A_{1}+A_{2}} H^{2}$ represents the baricentrical inertia of the two areas $\mathrm{A}_{1}$ and $\mathrm{A}_{2}$, supposed to be concentrated in the corresponding centroid.

For intermediate cases, Wölfel (1987) proposed a strong approximation according to which the effective moment of inertia is of the form

$$
I_{e q}=I_{1}+I_{2}+\Gamma \frac{A_{1} A_{2}}{A_{1}+A_{2}} H^{2},
$$


where the parameter $\Gamma, 0 \leq \Gamma \leq 1$, accounts for the capability of the interlayer to transfer shear stress between the glass plies. Wölfel proposed for $\Gamma$ the expression

$$
\Gamma=\frac{1}{1+\beta \frac{t E}{b l^{2}} \frac{A_{1} A_{2}}{A_{1}+A_{2}}},
$$

where the parameter $\beta$ depends upon the loading and boundary condition and, for the most common cases, the corresponding values are recorded in (Wölfel (1987)0. Hypothesis (3) is equivalent to assume that the individual bending stiffness of the external layers has no influence on the coupling offered by the central layer: the less the bending stiffness of the external layers, the more accurate is this hypothesis.

Bennison (2009) has adopted Wölfel's approach specifically for the case of laminated glass (Calderone et al. 2009). A strong approximation in their proposal consists in using in (3) the universal value $\beta=9.6$ although in Wölfel's theory this is associated to one case only, i.e., the case of simply supported beams under uniformly distributed load. From (3), one can easily calculate the stress- and the deflection-effective thickness, i.e., the (constant) thickness of the homogeneous plate that, under the same boundary and load conditions of the considered problem, has the same maximal stress or maximal deflection, respectively.

Introducing, as per (Bennison 2009), the quantities

$$
h_{s ; 1}=\frac{H h_{1}}{h_{1}+h_{2}}, h_{s ; 2}=\frac{H h_{2}}{h_{1}+h_{2}}, \quad I_{s}=\frac{1}{E} \frac{A_{1} A_{2}}{A_{1}+A_{2}} H^{2}=h_{1} h_{s ; 2}^{2}+h_{2} h_{s ; 1}^{2},
$$

the deflection-effective thicknesses turns out to be:

$$
h_{e f ; w}=\sqrt[3]{h_{1}^{3}+h_{2}^{3}+12 \Gamma I_{s}}
$$

whereas the stress-effective thickness for glass plies number 1 and 2 is given by

$$
h_{1 ; e f ; \sigma}=\sqrt{\frac{h_{e f ; w}^{3}}{h_{1}+2 \Gamma h_{s ; 2}}}, h_{2 ; e f ; \sigma}=\sqrt{\frac{h_{e f ; w}^{3}}{h_{2}+2 \Gamma h_{s ; 1}}} .
$$

Although these expressions (referred to in the sequel as the Wölfel-Bennison approach) refer to a very particular static scheme, they are commonly used in numerical computations with models of monolithic plates with constant thickness. The stress and strain so calculated are used for structural 
verification and, even more so, sometimes also serve to estimate stress concentrations around holes and/or at contact points; but no theoretical basis exists for this procedure.

An alternative formulation has been very recently proposed in (Galuppi and Royer-Cafagni 2012a). This procedure, called Enhanced Effective Thickness method, is based upon a variational approach and consists in finding the best approximation for the response of laminated glass among a restricted class of shape functions for the deflection surface through the minimization of the strain energy functional. The main hypotheses for this model are: $i$ ) the interlayer has no axial or bending stiffness, but only shear stiffness; ii) shear deformation of glass is neglected; iii) all materials are linear elastic; iv) geometric non-linearities are not considered. Remarkably, the method applies to the one-dimensional case of beams under bending (Galuppi and Royer-Cafagni 2012a) 0 but can be naturally extended to the two-dimensional case of plates (Galuppi and Royer-Cafagni 2012b) under the most various load and boundary conditions.

The purpose of this paper is to present the potentiality of this latter approach for the design of laminated glass. Paradigmatic cases are presented where its efficiency is proved by comparison with the results of precise numerical simulations and with the results obtainable with the classical Wölfel-Bennison approach. Tables for the calculation of the relevant coefficients in the most common cases have been added for ease of reference and to facilitate the practical use.

\section{Enhanced effective thickness approach}

The enhanced effective thickness (EET) method defines the equivalent moment of inertia $I_{R}$ as the weighted harmonic mean of the moments of inertia corresponding to the layered and monolithic limit. This is a substantial difference with respect to (3) that uses the weighted arithmetic mean. This approach can be applied to the most various static schemes and load conditions. 


\subsection{The one-dimensional case. Laminated glass beams.}

When applied to the same case of Figure 2, using the same notation of Section 1 the strain energy of the laminated beam can be written as a function of the vertical displacement $v(x)$, the same for the two glass components, and the horizontal displacements $u_{1}(x)$ and $u_{2}(x)$ of the centroid of the upper and lower glass ply, respectively. Under the hypothesis that strains are small and the rotations moderate, the minimization of the strain energy leads to differential equilibrium equations with appropriate boundary conditions, that can be hardly solved without the use of a numerical procedure.

In order to define simple expressions for the equivalent thickness, the problem is simplified by introducing convenient shape functions for $v(x), u_{1}(x)$ and $u_{2}(x)$ that are compatible with the qualitative properties of the solution. It is natural to consider as the shape function for $v(x)$ the form of the elastic curve $g(x)$ of a monolithic beam with constant cross section under the same loading and boundary conditions of the problem at hand. In particular, we set

$$
v(x)=\frac{g(x)}{E I_{R}},
$$

where $I_{R}$ is an unknown parameter representing the moment of the inertia of the laminated glass beam. We further assume that $I_{R}$ is the weighted harmonic mean of $I_{\text {tot }}$ (the monolithic limit) and $I_{1}+I_{2}$ (the layered limit), that is

$$
\frac{1}{I_{R}}=\frac{\eta}{I_{t o t}}+\frac{1-\eta}{I_{1}+I_{2}}
$$

where the non-dimensional weight parameter $\eta$ plays a role analogous to that of $\Gamma$ in (3), because it tunes the response from the layered limit $(\eta=0)$ to the monolithic limit $(\eta=1)$. As illustrated in (Galuppi and Royer-Cafagni 2012a), minimization of the strain energy allows to determine the best value of $\eta$ in the form 


$$
\eta=\frac{1}{1+\frac{E t}{G b} \frac{I_{1}+I_{2}}{I_{t o t}} \frac{A_{1}+A_{2}}{A_{1} A_{2}} \Psi},
$$

where, by denoting by $\Omega$ the one-dimensional domain representative of the reference configuration of the beam, the quantity $\Psi$ is defined as

$$
\Psi=\frac{\int_{\Omega} p(x) g(x) d x}{\int_{\Omega} g^{\prime}(x)^{2} d x}
$$

where $p(x)$ is associated with the distributed load (see Figure 2).

Clearly, $\Psi$ depends upon the boundary and load conditions and its values are recorded in Sect. 3 for the cases of most practical relevance. Notice as well that $\eta$ depends upon the mechanical and geometrical properties of the laminated beam, and one can show (Galuppi and Royer-Cafagni 2012) that when $G \rightarrow \infty$ then $\eta \rightarrow 1$ and when $G \rightarrow 0$, then $\eta \rightarrow 1$. From (9), the deflectioneffective thickness $\hat{h}_{w}$ then turns out to be

$$
\hat{h}_{w}=\sqrt{\frac{1}{\frac{\eta}{h_{1}^{3}+h_{2}^{3}+12 I_{s}}+\frac{1-\eta}{h_{1}^{3}+h_{2}^{3}}}} .
$$

Recalling the definitions (7) of $h_{s ; 1}$ and $h_{s ; 2}$, one also finds the following expressions for the stresseffective thickness:

$$
\hat{h}_{1 ; \sigma}=\sqrt{\frac{h_{1}^{3}+h_{2}^{3}+12 I_{s}}{2 \eta h_{s ; 2}}+\frac{\hat{h}_{w}^{3}}{h_{1}}}, \quad \hat{h}_{2 ; \sigma}=\sqrt{\frac{h_{1}^{3}+h_{2}^{3}+12 I_{s}}{2 \eta h_{s ; 1}}+\frac{\hat{h}_{w}^{3}}{h_{2}}} .
$$

The Enhanced Effective Thickness approach presents no additional difficulty with respect to the Wölfel-Bennison formulations, giving compact formulas (12) and (13) for laminated glass design. Moreover, it can be readily extended to the two-dimensional case.

\subsection{The two-dimensional case. Laminated glass plates.}


When considering the laminated glass plate identified by the $x-y$ domain $\Omega$ (see Figure 3 ) under distributed load $p(x, y)$, the strain energy can be written as a function of the vertical displacement $w(x, y)$, the same for the two glass plies, and the horizontal $x$ and $y$ components of displacements of the middle plane of the upper and lower glass plate. Minimization leads to a system of partial differential equations with appropriate boundary conditions. In order to simplify the problem, we again introduce a convenient shape functions for the displacement components.

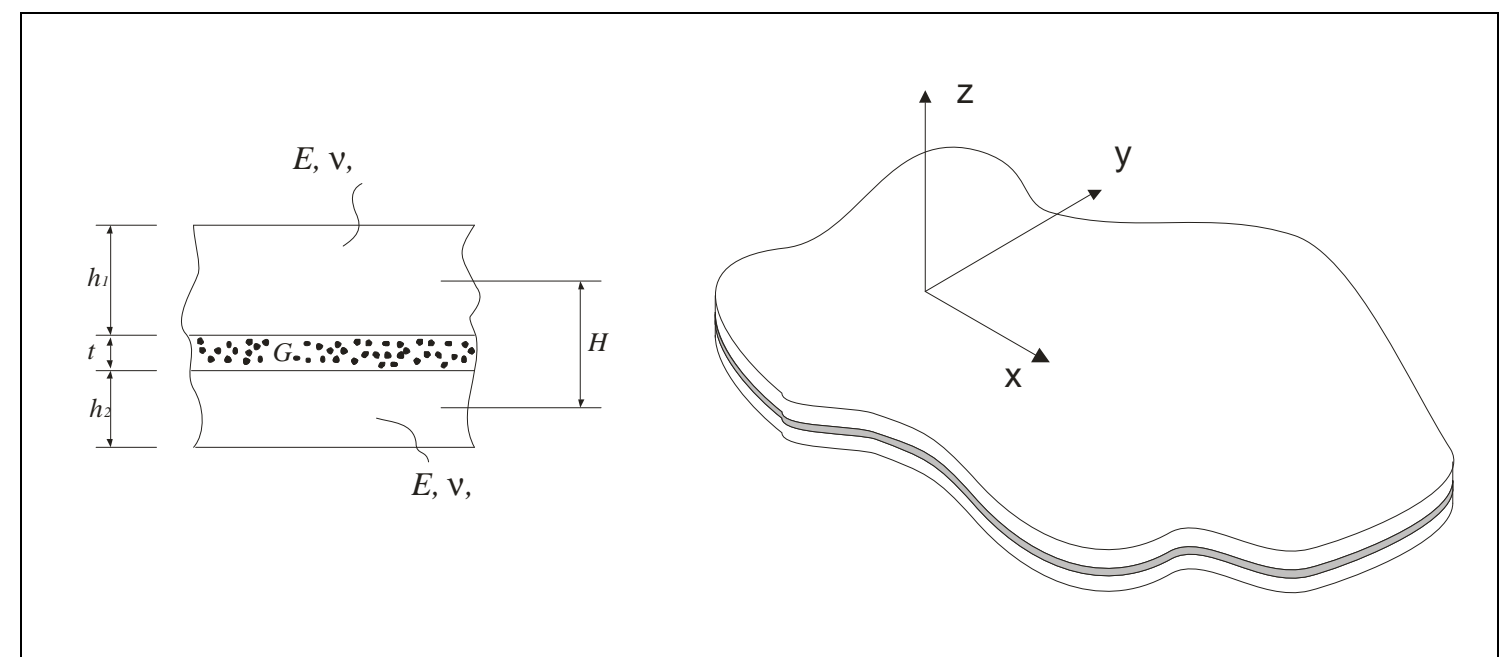

Figure 3: Plate composed of two glass plies bonded by a polymeric interlayer. Overall and cross sectional view (not in the same scale).

Defining the flexural rigidity of each glass ply as $D_{1}=\frac{E h_{1}^{3}}{12\left(1-v^{2}\right)}$ and $D_{2}=\frac{E h_{2}^{3}}{12\left(1-v^{2}\right)}$, it can be demonstrated that the flexural rigidity for the monolithic limit reads (Galuppi and Royer-Cafagni 2012b)

$$
D_{\text {tot }}=D_{1}+D_{2}+12 \frac{D_{1} D_{2}}{D_{1} h_{2}^{2}+D_{2} h_{1}^{2}} H^{2}
$$

Then, the shape function for $w(x, y)$ can be selected as the elastic deformed surface of a monolithic plate with constant thickness under the same loading and boundary conditions. In analogy with (5), we set

$$
w(x, y)=\frac{g(x, y)}{D_{R}}
$$


where $D_{R}$ is the equivalent rigidity and the shape function $g(x, y)$ is uniquely determined by the shape of the laminated glass plate in $x-y$ plane, by the external load $p(x, y)$ and by the geometric boundary conditions.

Assuming, in analogy with (9),

$$
\frac{1}{D_{R}}=\frac{\eta}{D_{t o t}}+\frac{1-\eta}{D_{1}+D_{2}},
$$

minimization of the strain energy allows to determine the counter part of (10) for the two dimensional case in the form

$$
\eta=\frac{1}{1+\frac{t}{G} \frac{D_{1}+D_{2}}{D_{t o t}} \frac{12 D_{1} D_{2}}{D_{1} h_{2}^{2}+D_{2} h_{1}^{2}} \Psi},
$$

where now

$$
\Psi=\frac{\int_{\Omega} p(x, y) g(x, y) d x d y}{\int_{\Omega}\left[g_{, x}(x, y)^{2}+g_{, y}(x, y)^{2}\right] d x d y},
$$

depends upon the plate shape, the load distribution $p(x, y)$ and the boundary conditions. The stressand deflection-effective thicknesses may be readily calculated and take expressions analogous to (12) and (13), respectively.

It is important to note that the only "difficulty" of the proposed method consists in calculating $\Psi$ from (18), because all the other formulas are simple analytical expressions. In the following we will report tables with values of $\Psi$ that refer to the most common cases of the design practice.

\section{Examples}

The results obtainable with the EET approach are now compared with those proposed by Bennison (2009) and with the numerical experiments performed by means of the finite element software SJ-Mepla, specifically conceived of for laminated glass (SJ MEPLA 2011).

\subsection{One-dimensional examples. Various constraint and load conditions.}


Table 1 summarizes the values of $\Psi$ evaluated through equation (11) as a function of the beam length $l$ for various constraint and load condition. Such a coefficient allows to simply evaluate $\eta$ through equation (10)

\begin{tabular}{|c|c|c|c|}
\hline $\begin{array}{l}\text { LOADING AND BOUNDARY } \\
\text { CONDITIONS }\end{array}$ & $\Psi$ & $\begin{array}{l}\text { LOADING AND BOUNDARY } \\
\text { CONDITIONS }\end{array}$ & $\Psi$ \\
\hline$\frac{d}{k}$ & $\frac{168}{17 l^{2}}$ & $\hat{\kappa}$ & $\frac{42}{l^{2}}$ \\
\hline$\frac{b}{l}$ & $\frac{15}{l^{2}+2 a b}$ & 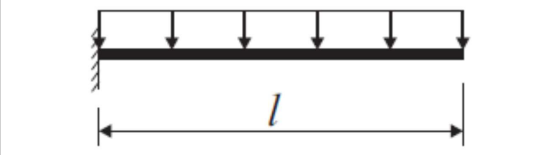 & $\frac{14}{5 l^{2}}$ \\
\hline$l / 2$ & $\frac{10}{l^{2}}$ & 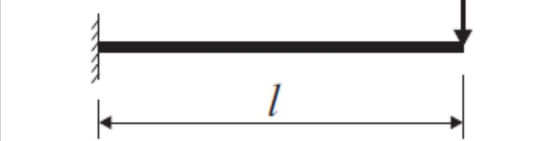 & $\frac{5}{2 l^{2}}$ \\
\hline 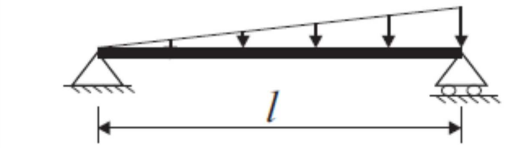 & $\frac{10}{l^{2}}$ & $\underset{1}{\longrightarrow}$ & $\frac{45}{14 l^{2}}$ \\
\hline 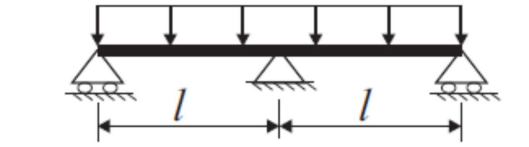 & $\frac{21}{l^{2}}$ & 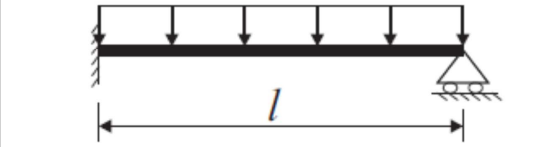 & $\frac{21}{l^{2}}$ \\
\hline
\end{tabular}

Table 1 : Laminated glass beams under different boundary and load conditions; values of coefficient $\Psi$ for different boundary and load conditions.

For the sake of comparison, in the present section, four paradigmatic cases are analyzed in detail. With the same notation of Figure 2, assumed geometrical and structural parameters are $l=3150$ $\mathrm{mm}, b=1000 \mathrm{~mm}, h_{1}=h_{2}=10 \mathrm{~mm}, t=0.76 \mathrm{~mm}, E=70 \mathrm{GPa}$, while the shear modulus $G$ of the polymeric interlayer is varied to evaluate its influence on the shear-coupling of the glass plies. The distributed pressure on the beam is taken equal to $0.75 \mathrm{kN} / \mathrm{m}^{2}$ so that, with $b=1000 \mathrm{~mm}$, the distributed load per unit length becomes $p=0.75 \mathrm{~N} / \mathrm{m}$. For the case of concentrated force, we take $F=1 \mathrm{kN}$

In the following graphs, the stress- and deflection-effective thicknesses, calculated through (12) and (13), are plotted as function of $G$ with a continuous line, whereas the effective thicknesses 
calculated with the Wölfel-Bennison's is represented with a dashed curve. Results of numerical experiments are indicated with dots.

The cases considered here are: $i$ ) simply supported beam under uniformly distributed load (

Figure 4); ii) simply supported beam under concentrated load (Figure 5); iii) beam with three supports under distributed load (Figure 6); iv) double clamped beam under uniformly distributed load (Figure 7). For case ii), the value $\beta=12$, recorded in the original Wölfel paper (Wölfel 1987) has been used.
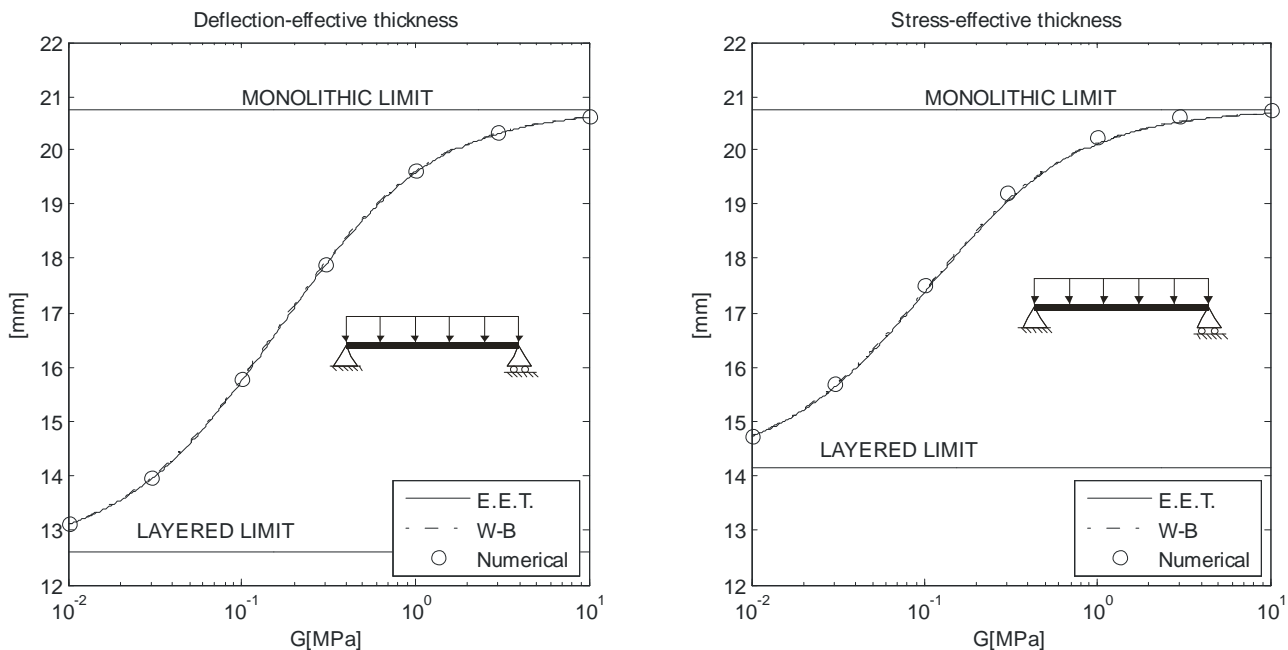

Figure 4: Simply supported beam under uniform load. Comparison of the effective thicknesses obtained with: Wölfel-Bennison (WB) approach; the enhanced effective thickness (EET) approach; the numerical simulations.
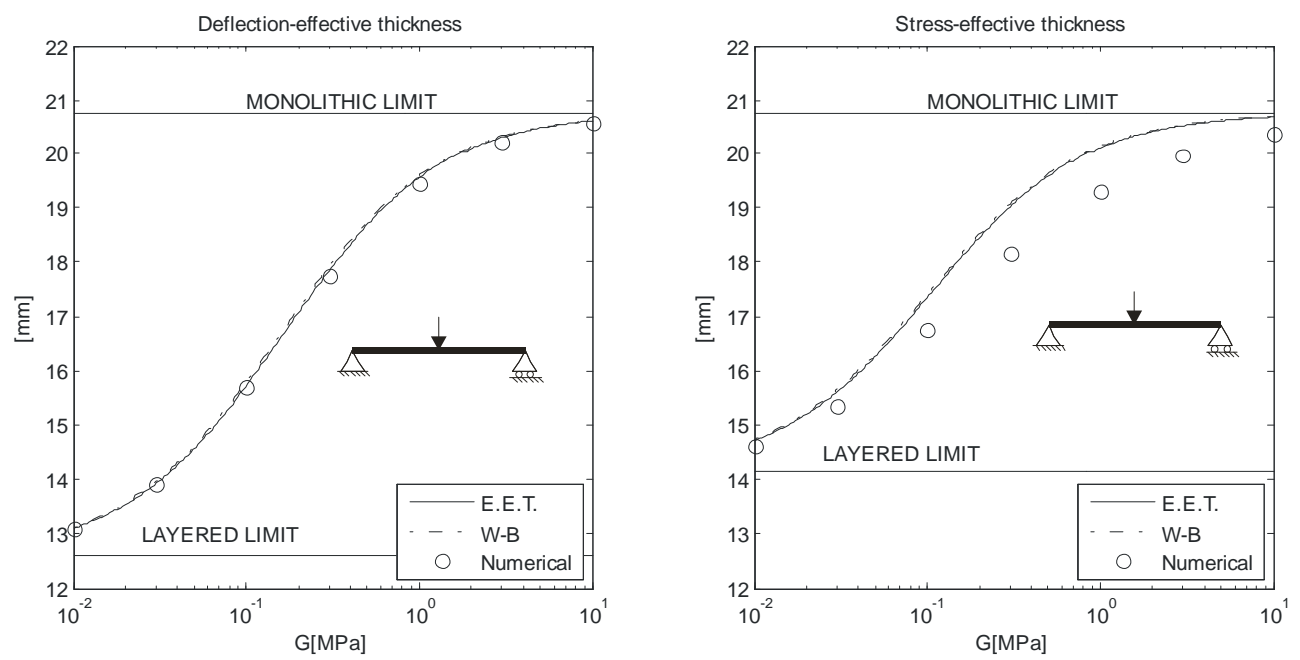

Figure 5: Simply supported beam under concentrated load. Comparison of the effective thicknesses obtained with: Wölfel-Bennison (WB) approach with $\boldsymbol{\beta}=\mathbf{1 2}$; the enhanced effective thickness (EET) approach; the numerical simulations. 

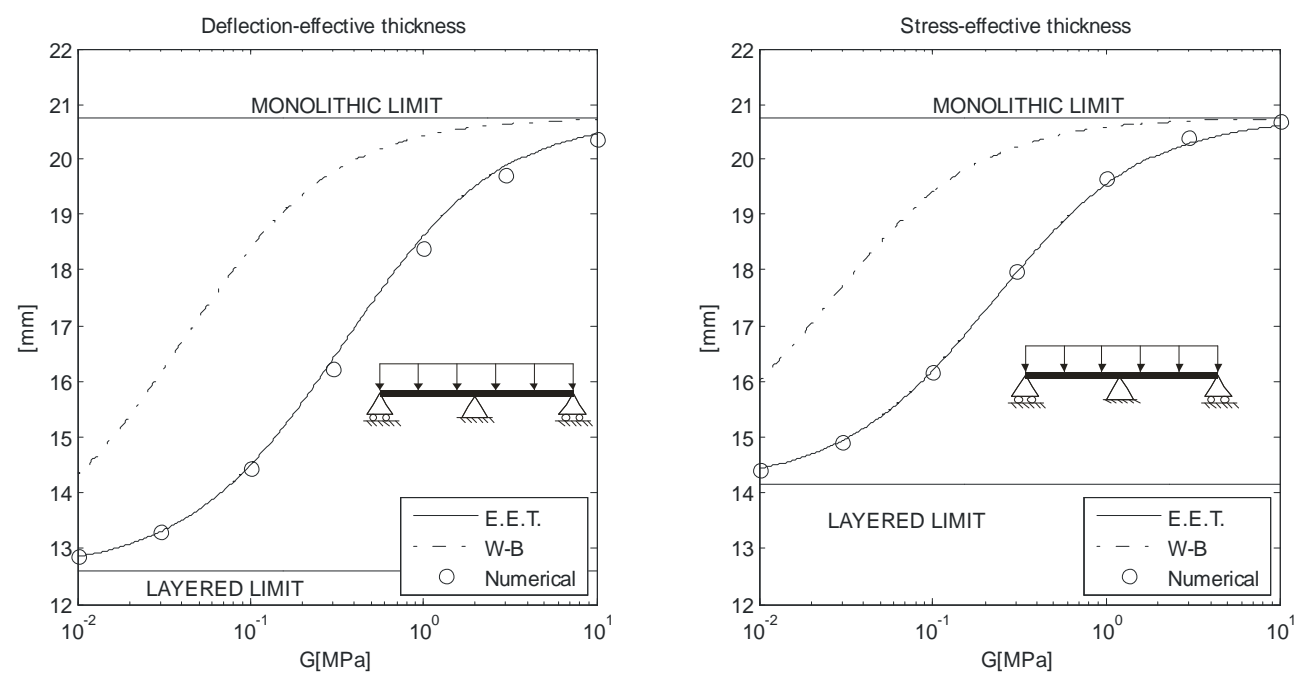

Figure 6: Beam with three supports under distributed load. Comparison of the effective thicknesses obtained with: Wölfel-Bennison (WB) approach; the enhanced effective thickness (EET) approach; the numerical simulations.
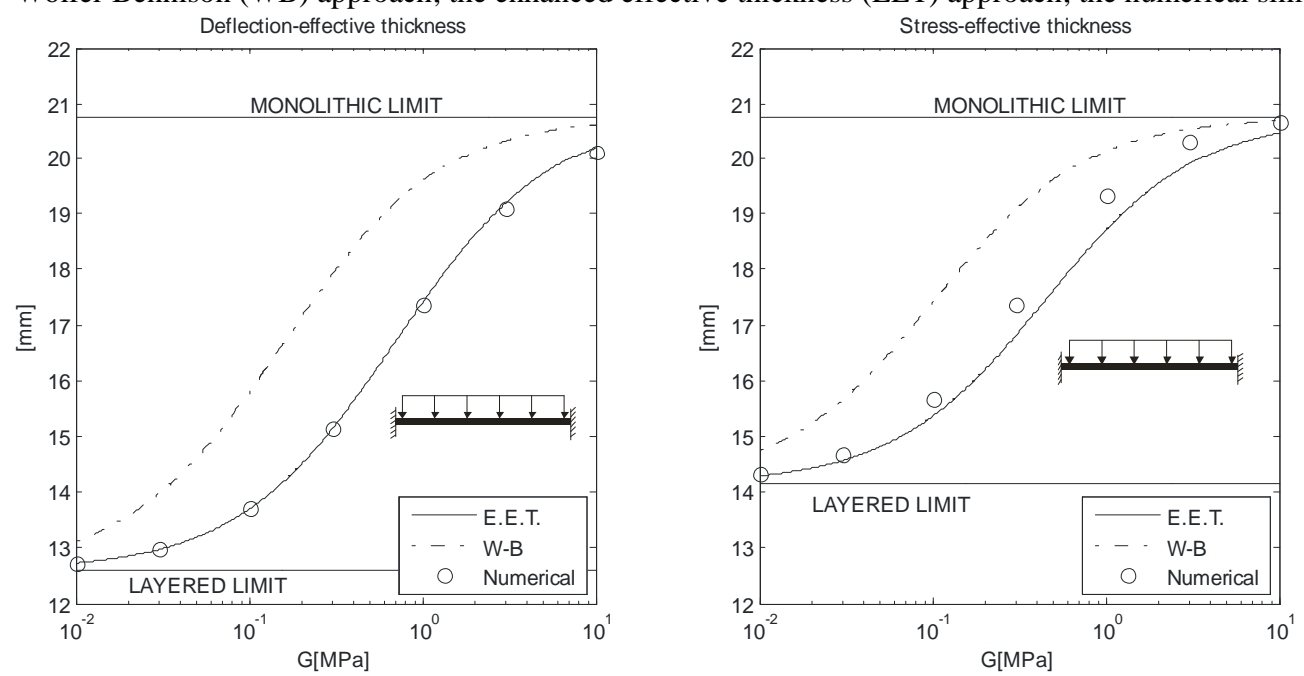

Figure 7: Double clamped beam under distributed load. Comparison of the effective thicknesses obtained with: Wölfel-Bennison (WB) approach; the enhanced effective thickness (EET) approach; the numerical simulations.

In the case of simply supported beams under uniform load the models give results that in practice coincide, a finding that is not surprising because this is the simplest case upon which the Wölfel approach is calibrated. Numerical results confirm the good approximation that is achieved. Also for the case of simply supported beam under concentrated load, the two approaches give results that practically coincide. However, it is evident from Figure 5 that the agreement with the numerical simulations is good for the deflection effective thickness, whereas the stress-effective thickness is qualitatively different, especially in those branches close to the monolithic limit. 
In the case of beam with three supports and of clamped beam, there is a substantial deviation between the EET and W-B approaches especially for the lowest values of $G$, but the numerical experiments are in favor of the EET approach. Observe that W-B is not on the side of safeness, because it predicts effective thicknesses greater than in reality and, consequently, underestimates deflection and stress.

\subsection{Two-dimensional examples. Plates under various constraint and load conditions}

In the present section, several cases of practical importance for rectangular plates are analysed. Apart from uniformly distributed pressure, we have also considered the action of a (pseudo-) concentrated load whose imprint, according to the indication of most structural standards. is supposed to be a $100 \mathrm{~mm} \times 100 \mathrm{~mm}$ square.

Tables 2.1 and 2.2 collect values of the coefficient $\Psi\left[\mathrm{mm}^{-2} \cdot 10^{6}\right]$ that are necessary to evaluate $\eta$ as per (17), calculated according to equation (18) as a function of the plate length $a[\mathrm{~mm}]$ and of the aspect ratio $\lambda=b / a$.

The shape functions $g(x, y)$ for $w(x, y)$, introduced in (15), can be found in (Timoshenko 1970) and (Batista 2010) in the form of trigonometric and hyperbolic series. In the calculation of $\Psi$ as per (18), we have considered only the first term in the series (first order approximation) for the cases in which the load is distributed; it can be directly verified that higher order approximations, obtained by considering more terms of the series, do not substantially increase the level of accuracy. On the other hand, when the plate is loaded on a small area (pseudo-concentrated load), the use of higher-order terms of the series increases notably the precision of the deflection- and stress-effective thickness. In Table 2.1, the values of $\Psi$ for plates under pseudo-concentrated load have been obtained by using a third order approximation.

It should also be remarked that for the case of plates with one edge built in, the deformed shape under a uniformly distributed load is cylindrical in type and, consequently, the coefficient $\Psi$, 
and hence the coefficient $\eta$ and the deflection- and stress-effective thickness, turns out to be independent upon the width $b$.

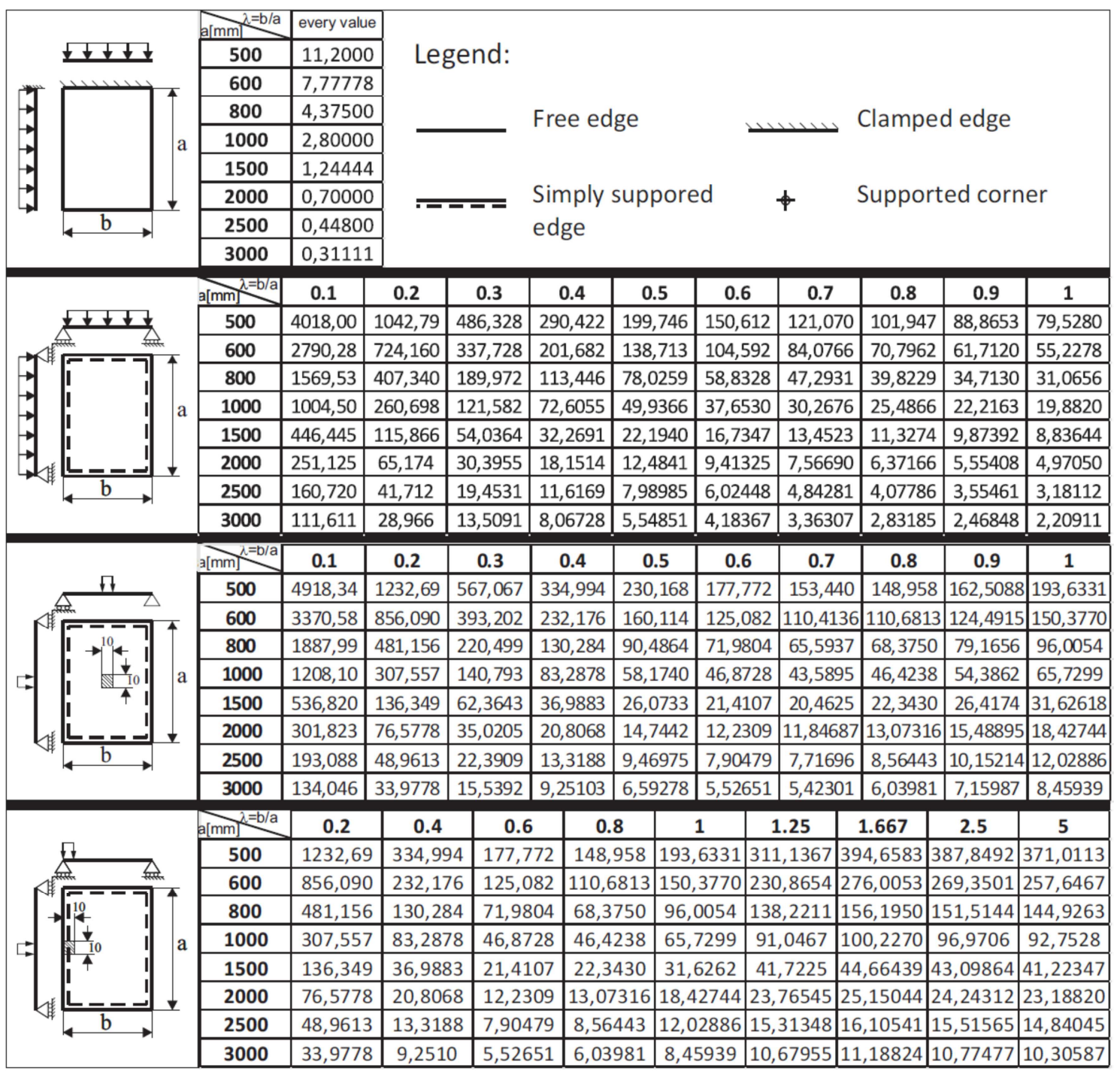

Table 2.1: Representative examples of laminated glass plates: values of coefficient $\Psi\left[\mathrm{mm}^{-2} \cdot 10^{6}\right]$ for different load and boundary conditions. 


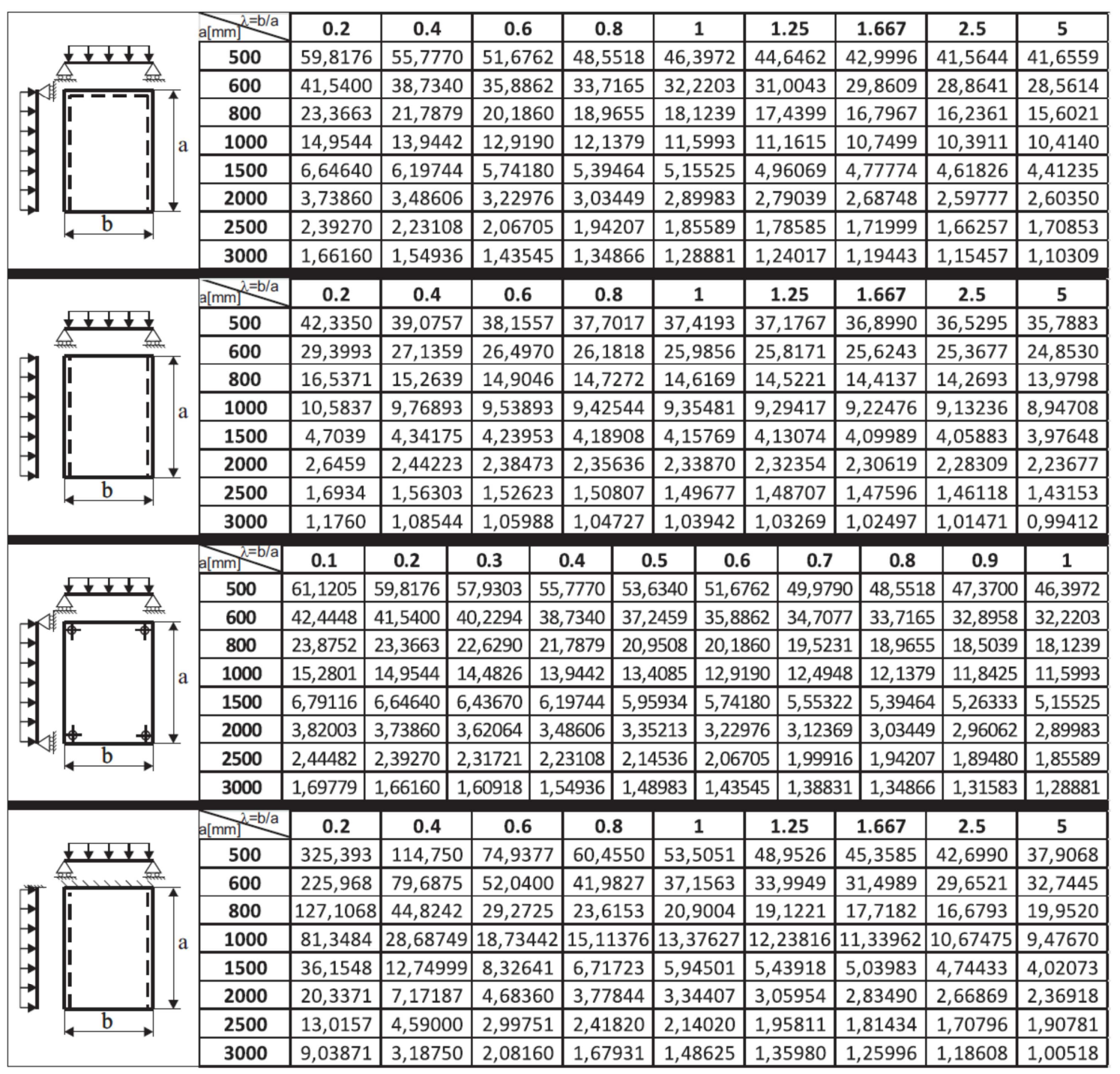

Table 2.2: Further examples of laminated glass plates under various boundary and load conditions (same notation of Table 2.1). Value of the coefficient $\Psi\left[\mathrm{mm}^{2} \cdot 10^{6}\right]$.

It is important to note that:

- for plates with the same boundary and loading condition in $x$ and $y$ direction (for example plate supported on four sides) under a constant distributed load, the parameter $a$ denotes the longer edge of the plate (note that, in such cases, Tables 2.1 and 2.2 give $\lambda=b / a<1$ );

- for plates with different boundary and loading condition in $x$ and $y$ direction (for example plate supported on two sides) under a constant distributed load, the identification of the edges is shown in the sketch of Tables 2.1 and 2.2; in such cases Tables give either $\lambda<1$ or $\lambda>1$. 
For example, the value of coefficient $\Psi$ for a plate of dimension $3000 \mathrm{~mm} \times 1800 \mathrm{~mm}$, supported on $3000 \mathrm{~mm}$ edge, can be found in table 2.2 by choosing $a=3000 \mathrm{~mm}, \lambda=0.6$.

In the sequel, we compare the deflection- and stress-effective thickness calculated according to the proposed EET approach through equations (12) and (13), with the ones calculated with the W-B formulas (3) and (4). Results are also validated by means of numerical analysis performed by the finite element software SJ-Mepla. Assumed structural parameters are the size of the plate $a=$ $3000 \mathrm{~mm}$ and $b=2000 \mathrm{~mm}$; the thicknesses of the glass plies $h_{1}=h_{2}=10 \mathrm{~mm}$; the thickness of the interlayer $t=0.76 \mathrm{~mm}$; the elastic parameters for glass $E=70 \mathrm{GPa}$ and $v=0.22$. The shear elastic modulus $G$ of the polymeric interlayer is again varied between $0.01 \mathrm{MPa}$ and $10 \mathrm{MPa}$. The distributed pressure on the plate is taken equal to $0.75 \cdot 10^{-3} \mathrm{~N} / \mathrm{mm}^{2}$.

The most frequent case in the design practice is certainly that of a rectangular plate with all the sides simply supported, subject either to a distributed or concentrated load. The graphs of Figure 8 compare the deflection- and stress-effective thickness calculated according to the EET and the W-B approaches to the results of the numerical experiments. It is very evident here that the two formulations give different results at the qualitative level. Again W-B is not on the side of safeness, because it underestimate deflection and stress.
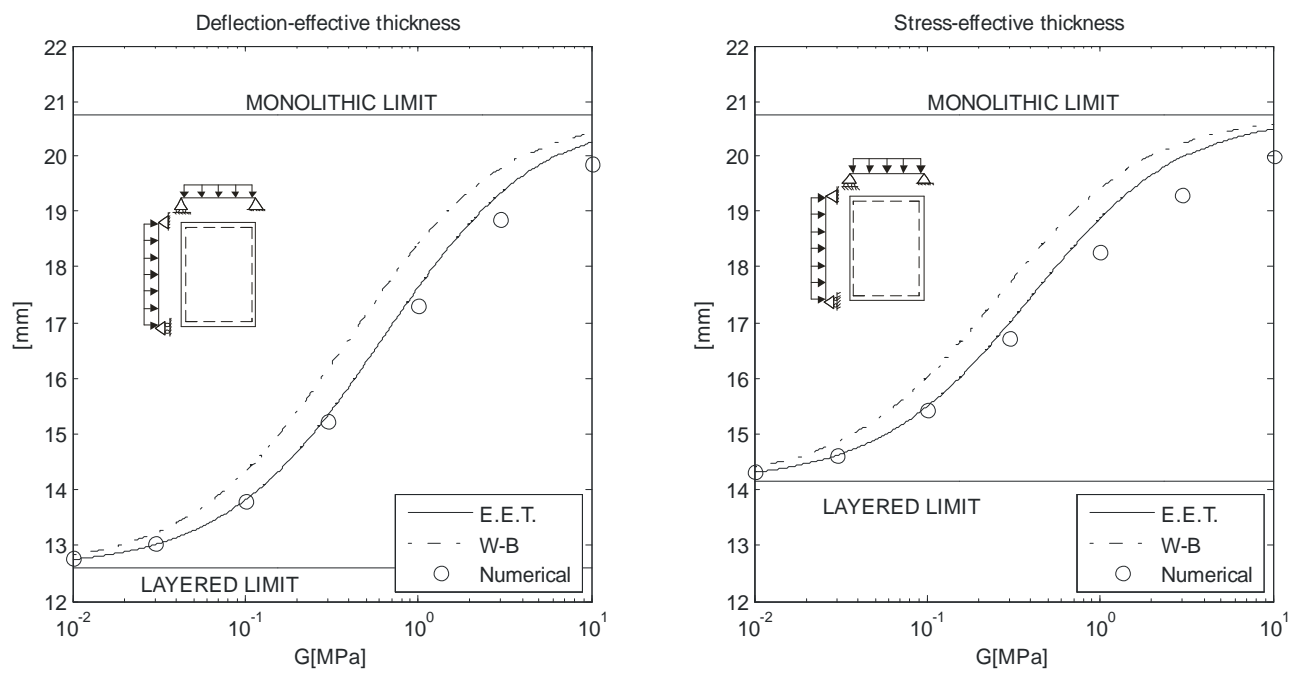

Figure 8: Rectangular plate simply supported on four sides under distributed load. Comparison of the effective thicknesses obtained with: Wölfel-Bennison (WB) approach; the enhanced effective thickness (EET) approach; the numerical simulations. 
In such a case, the behavior predicted by the EET approach is close to Wölfel-Bennison's whenever the aspect ratio is such that plate response is similar to the response of a beam $(\lambda=b / a \gg 1)$. This is not surprising because the Wölfel-Bennison's model is calibrated on the case of simply supported beams under uniformly distributed load. On the contrary, the greatest differences between the EET and W-B approaches are obtained when the plate is square $(\lambda=1)$, i.e., when the deflections of beam and plate differ the most. This is shown in Figure 9, where the percentage error on the evaluation of the deflection- and stress- effective thicknesses are plotted as a function of the aspect ratio $\lambda$.
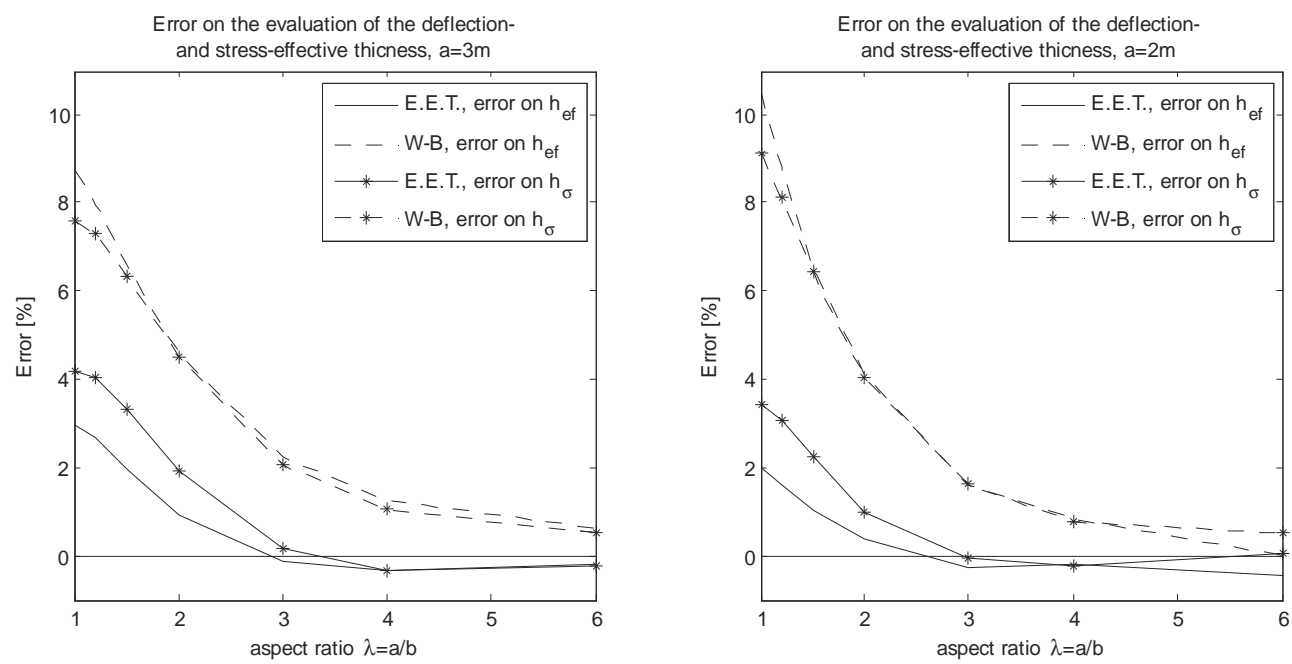

Figure 9: Error on the evaluation of the deflection- and stress-effective thickness, for different plate lengths $a$ and aspect ratio.

In the case of rectangular plates simply supported on four sides under a pseudo-concentrated load, the conclusions about the stress and deflection-effective thicknesses are similar to those for the case of uniformly distributed load. As mentioned above, under load conditions of this type, consideration of just the first-order approximation of the shape function $g(x, y)$ does not give acceptable accuracy. This finding is evidenced in Figures 10 and 11, where a comparison is made between the effective thicknesses evaluated with either first-order approximation or third-order approximation. It is evident from the graphs that the use of third-order terms in the series improves the precision especially for what the calculation of deflection is concerned. 
For such cases, the value $\beta=9.6$ proposed by Bennison has been used; the value $\beta=12$ recorded in the original work by Wölfel for a beam under concentrated load does not lead to better results.
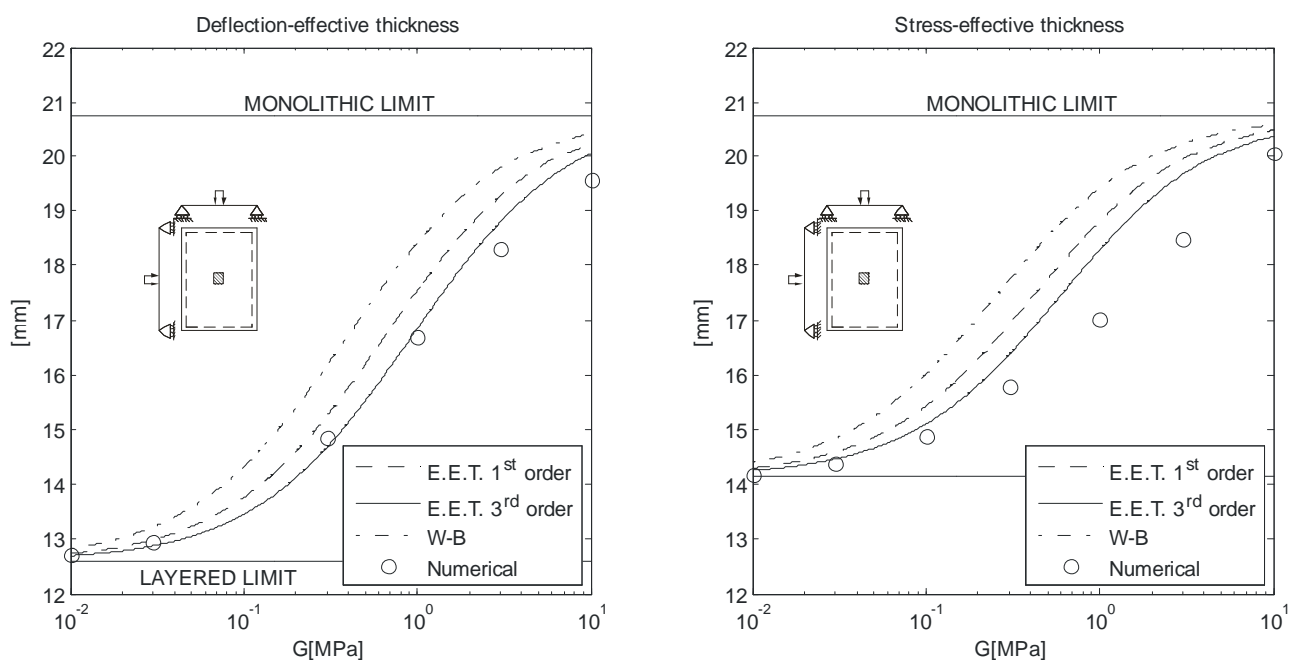

Figure 10 Rectangular plate simply supported on four sides under pseudo-concentrated load acting at the centre of the plate. Comparison of the effective thicknesses obtained with: Wölfel-Bennison (WB) approach; the enhanced effective thickness (EET) approach $\left(1^{\text {st }}\right.$ and $3^{\text {rd }}$ order accuracy); the numerical simulations.
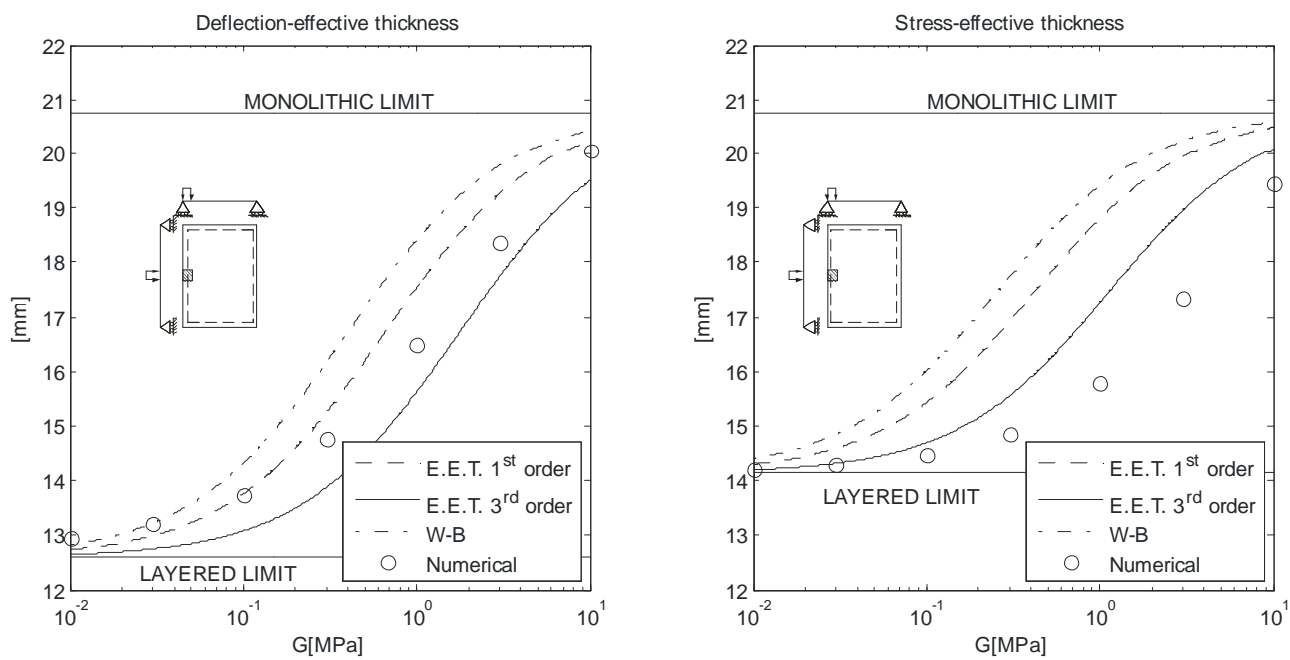

Figure 11 Rectangular plate simply supported on four sides under concentrated load acting at the middle of one edge of the plate. Comparison of the effective thicknesses obtained with: Wölfel-Bennison (WB) approach; the enhanced effective thickness (EET) approach $\left(1^{\text {st }}\right.$ and $3^{\text {rd }}$ order accuracy); the numerical simulations.

Figure 12 compares the EET and W-B results for the case of plate simply supported on three sides (with a free side of length $b$ ). In this condition both models give results in good agreement with the numerical experiments. 

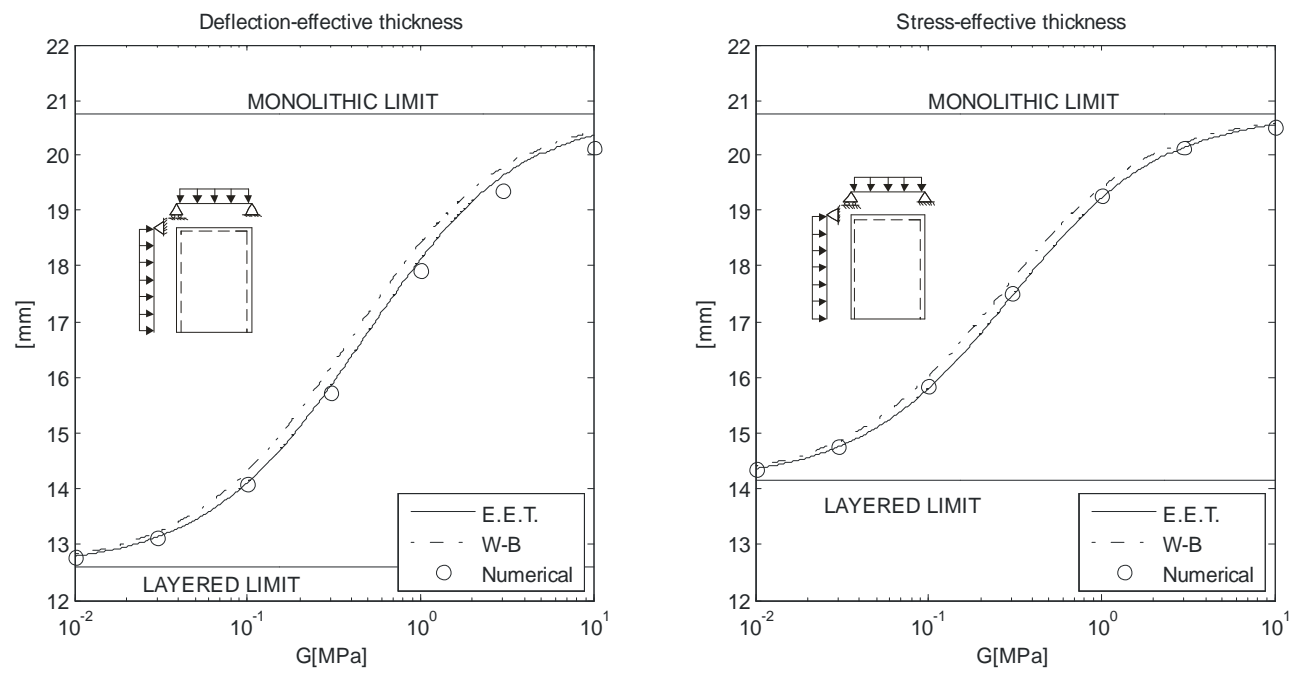

Figure 12: Rectangular plate simply supported on three sides under distributed load. Comparison of the effective thicknesses obtained with: Wölfel-Bennison (WB) approach; the EET approach; the numerical simulations.

It should also be observed, as discussed at length in (Galuppi and Royer-Cafagni 2012b), that when the deformation of the plate tends to be cylindrical, so that its response is similar to that of a beam, the predictions of W-B and EET tend to coincide. This is the case of a plate simply supported on two opposite sides, to which Figure 13 refers to.
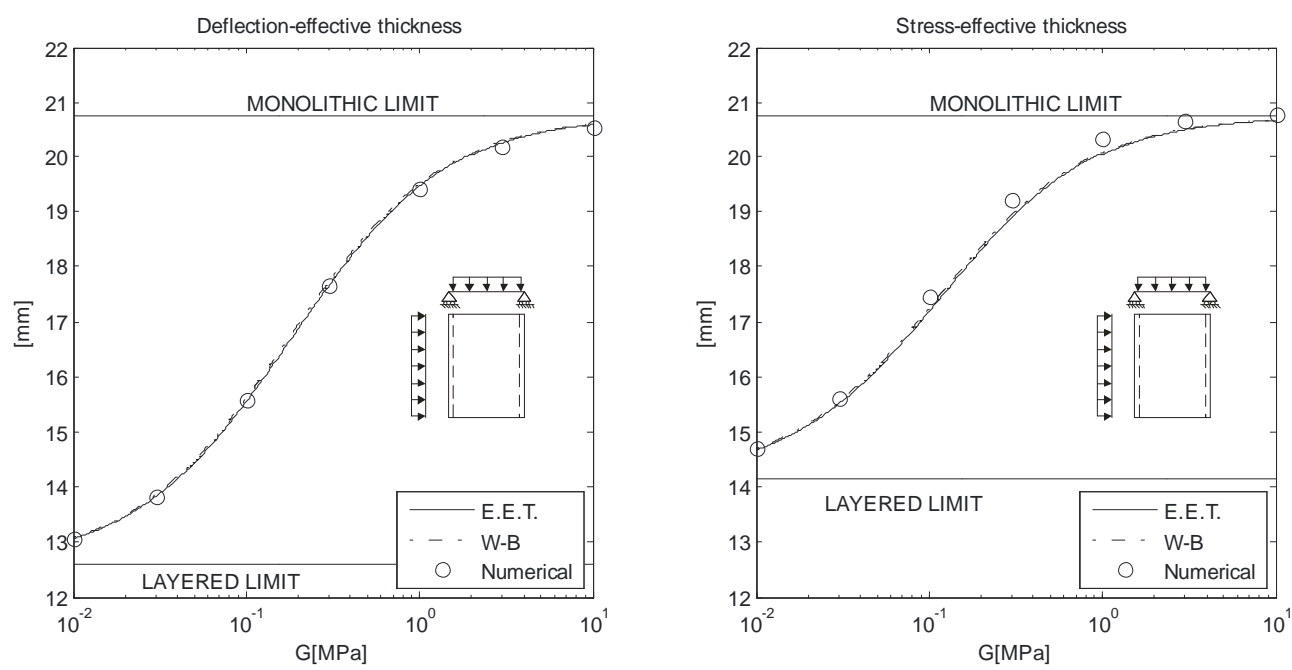

Figure 13: Rectangular plate simply supported on two sides under distributed load. Comparison of the effective thicknesses obtained with: Wölfel-Bennison (WB) approach; the EET approach; the numerical simulations.

The case of rectangular plates point-wise supported at the corners does apply to frameless glazing. It is evident from Figure 14 that the EET and W-B give similar results, in agreement to numerical outcomes. 

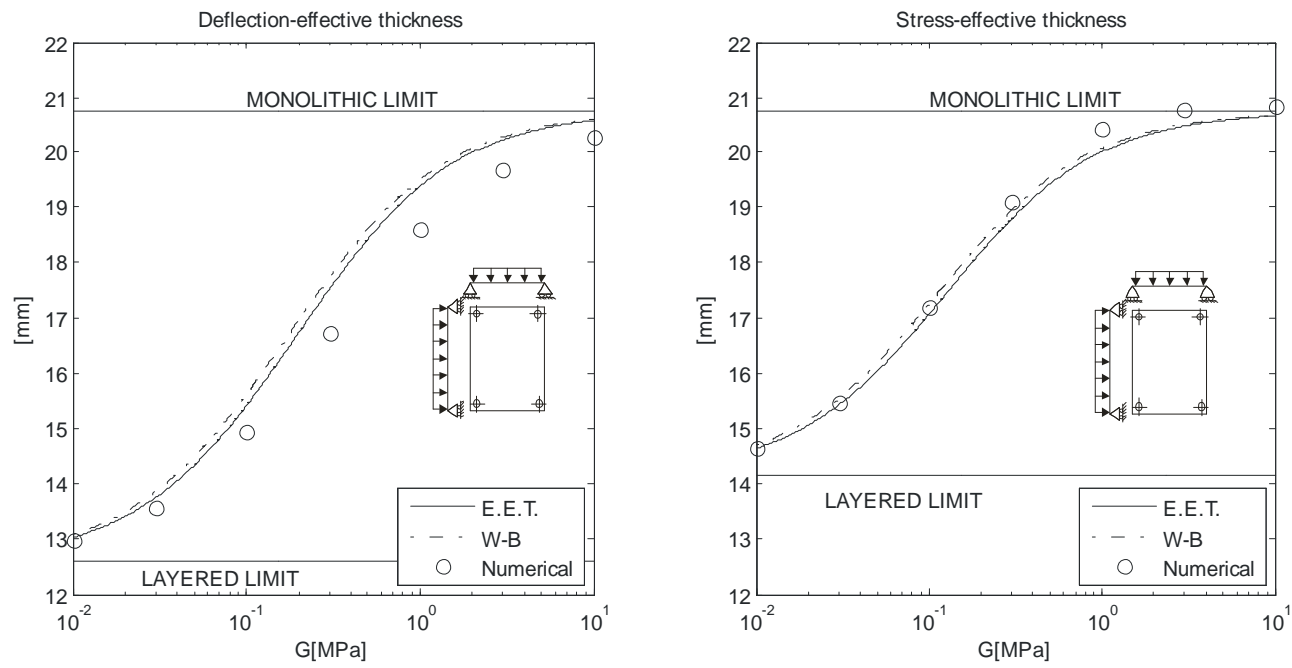

Figure 14: Rectangular plate simply supported at the four corners under distributed load. Comparison of the effective thicknesses obtained with: Wölfel-Bennison (WB) approach; the enhanced effective thickness (EET) approach; the numerical simulations.

In the case of rectangular plate with two opposite edge simply supported, the third edge built in and the fourth edge free, it is evident from Figure 15 that the Enhanced Effective Thickness model and Wölfel-Bennison approach give substantially different results and that numerical experiments are in favor of EET.
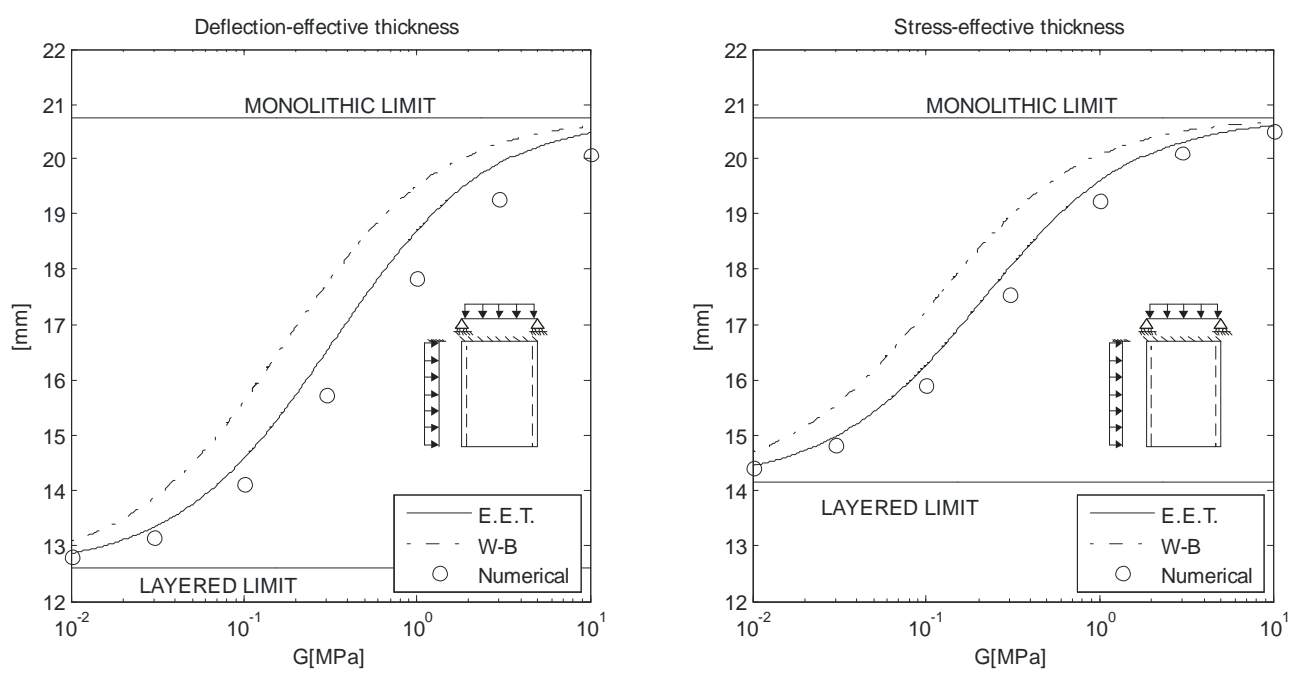

Figure 15: Rectangular plate with two opposite edges simply supported and one edge built in, under distributed load. Comparison of the effective thicknesses obtained with: Wölfel-Bennison (WB) approach; the enhanced effective thickness (EET) approach; the numerical simulations.

Figure 16 shows the comparison the EET and W-B results for the case of rectangular plate with one edge built-in. From this, it is evident that the EET and W-B now give substantially different 
results; deflection-effective thickness calculated through EET approach is in agreement with the results of the numerical simulation. The evaluation of the stress-effective thickness is not so precise, because it is affected by stress intensification near the clamped edge.
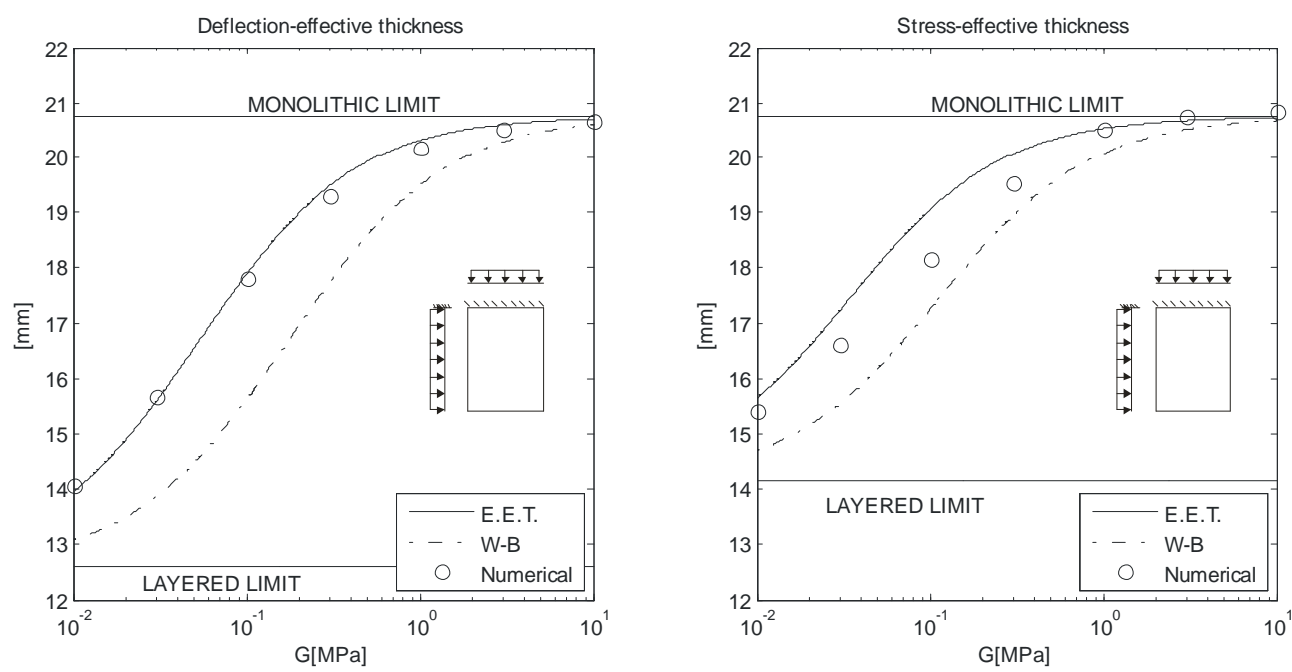

Figure 16 Rectangular plate with one edge built in under distributed load. Comparison of the effective thicknesses obtained with: Wölfel-Bennison (WB) approach; the EET approach; the numerical simulations.

\section{Conclusions}

One of the currently most-used simplified approaches for the structural design of laminated glass is that due to Bennison (2009), which is based upon the original work by Wölfel (1987). However, Wölfel's model was primarily conceived of for a sandwich beam with external plies with considerable axial stiffness but negligible bending stiffness and an intermediate layer that can only bear shear stress, with zero axial and flexural strength. Whenever the external layers present considerable bending stiffness, as in the case of laminated glass, Wölfel proposed a very approximate solution that in any case, as we have verified here, gives results in agreement with more accurate (numerical) methods of analysis for the only case in which the load is uniformly distributed and the deformed shape tends to be cylindrical, i.e. case of simply supported beams or rectangular plates simply supported on two opposite sides.

When the load is not uniformly distributed, the standard Wölfel - Bennison approach gives results that are not on the side of safeness. Better approximations can be achieved with the 
Enhanced Effective Thickness approach. Here we have recorded the significant parameters necessary for a quick calculation of the effective thickness for the cases of most practical importance, which presents no additional difficulty with respect to the more traditional formulation.

In the two-dimensional case of plates, the results obtained with Wölfel-Bennison are accurate only when the plate is rectangular and simply supported on two opposite edges, i.e., when its deformed shape tends to be cylindrical and its response similar to that of a simply supported beam. When this is not the case, the Enhanced Effective Thickness method gives results that fit more closely the real situation both for the deflection and the stress calculation.

The EET method furnishes compact formulas also for the two-dimensional case and, remarkably, the most relevant expression (12) and (13) are analogous to those corresponding to the one dimensional case. The coupling offered by the interlayer can be readily evaluated by using the values of $\Psi$ that have been tabulated here for all those cases that are relevant for the design practice. However, using (18), the value of $\Psi$ can be calculated with no difficulty for any laminated plate under any load condition. The enhanced effective-thickness approach thus seems to represent an accurate and powerful tool for the practical calculation of laminated glass.

\section{Acnowledgement.}

The autors acknowledge the Italian MURST for its partial support under the PRIN2008 program.

\section{REFERENCES}

Batista, M. (2010). "New analytical solution for bending problems of uniformly loaded rectangular plate supported on corner points", IES J. Part A: Civ. Struct. Eng., 3, 75-84.

Bennison, S.J. (2009) "Structural properties of laminated glass", Short Course, Glass Performance Days, Tampere, Finland. 
Calderone, I., Davies, P.S., Bennison, S.J., Huang, X. Gang, L. (2009). "Effective laminate thickness for the design of laminated glass", Proc., Glass Performance Days, Tampere, Finland.

Galuppi, L., Royer-Carfagni, G., (2012a). "Effective Thickness of Laminated Glass Beams. New expression via a Variational Approach", Eng. Struct., in press. Available on line at http://dspaceunipr.cilea.it/handle/1889/1702

Galuppi, L., Royer-Carfagni, G., (2012b). "The effective thickness of laminated glass plates", submitted. Available on line at http://dspace-unipr.cilea.it/handle/1889/1703

Newmark, N.M., Siess, C.P., and Viest, I.M. (1951). "Test and analysis of composite beams with incomplete interaction.” Proc. Soc. for Experimental Stress Analysis, 9, 75-92.

SJ MEPLA (2012), “User’s manual”, version 3.5.

Timoshenko, S.P., Woinowsky-Krieger, S. (1970). Theory of Plates and Shells , McGraw Hill

Wölfel, E., (1987). "Nachgiebiger Verbund Eine Näherungslösung und deren Anwendungsmöglichkeiten.” Stahlbau, 6, 173-180. 